\title{
A Study of the Applicability of Different Types of Interatomic Potentials to Compute Elastic Properties of Metals with Molecular Dynamics Methods
}

\author{
Nadezhda Chistyakova ${ }^{\text {a)}}$, Thi My Hue Tran ${ }^{\text {b) }}$ \\ Tomsk Polytechnic University, 30 Lenina Avenue, Tomsk 634050 Russian Federation \\ a) corresponding author: chistyakovanv@tpu.ru \\ b) myhuesp2@gmail.com
}

\begin{abstract}
The paper describes three common types of interatomic interaction potentials used for constructing theoretical models of the matter. The pair potentials are the Morse potential and the multiparticle potentials are the embedded atom method (EAM), the modified embedded atom method (MEAM). The rules of potential constructing and the fields of their application have been considered. Three types of potentials was used for calculation the elastic properties of palladium. The aim of the work is to determine which of the potentials - Morse potential, EAM or MEAM are better suited for calculating properties of palladium. It was found that all three potential approximately equally determine the properties of palladium. However, the Morse potential has the advantage because its structure is much simpler.
\end{abstract}

\section{INTRODUCTION}

Interatomic potentials are used for describing interaction between atoms in a matter. Such description is very important for developing theoretical models of the matter. These models are used for predicting characteristics of the matter and describing the matter behavior in different conditions [1-2].

The potential is usually a functional dependence of potential atomic energy from distance in a space. The simplest example of a field is the electrostatic field of point charges. Figure 1 shows the distribution of the total potential field created by four identical point charges. The interaction energy between atoms is known to be the energy of the electric field created by electrons and atomic nuclei (Fig.2). Nuclei and electrons distribution defines the quantity of field in the different points of space.

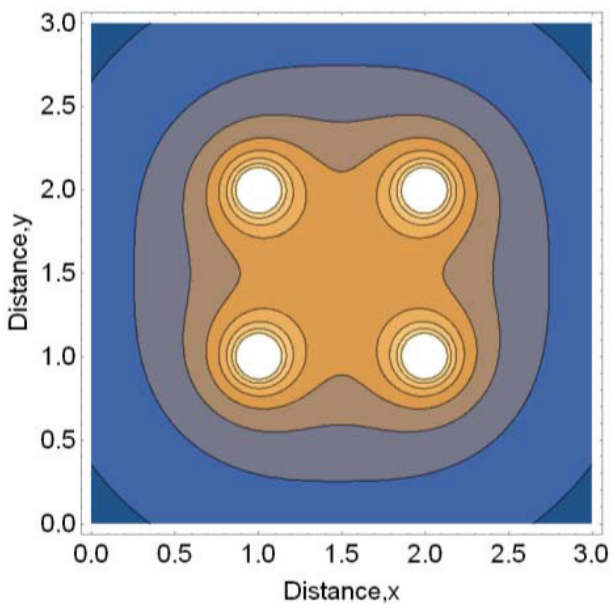

FIGURE 1. The electrostatic field of system with four equal charges [3]

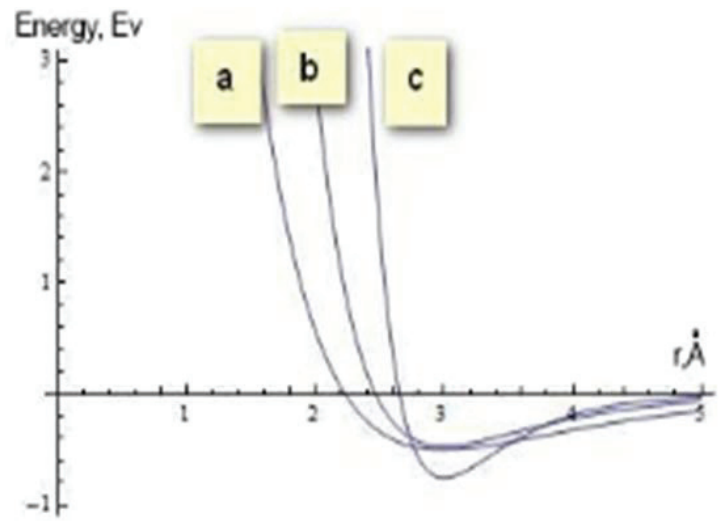

FIGURE 2. The Morse potentials for different pairs of atoms: Pd-Pd (a), H-H (b), Pd-H (c) [4] 
In 1927 Heitler and London calculated energy for a system consisting from two hydrogen atoms [5]. The result of this calculation has shown that there is the repulsive force between atoms at the short distance. The attractive force appears at the longer distance. The repulsive force is explained by the Pauli principle. The attractive force has more complicated quantum nature because of charge distribution.

There are different methods for potential defining. The simplest way is the pair potential method. This method determines the energy of atom as the sum of interaction energy between all atomic pairs. However, this type of methods does not take into account the influence of neighboring atoms on pair interaction, although neighboring atoms could have a significant effect.

Well-known pair potentials include Lennard-Jones potential, Morse potential and others. These potentials are different empirical functions from distance. Empirical function means that structure and parameters of functions are obtained from experiment not from theory.

The effect with neighboring atoms is considered in such types of potentials as multiparticle potentials. The most common among them is embedded atom method (EAM) and its advanced version is modified embedded atom method (MEAM). These methods are semiempirical, because their formalism uses some theoretical conceptions and experimental data.

This paper will deal only with EAM and MEAM because of their wide distribution. Beside empirical and semiempirical potentials there are potentials obtained by ab-initio (first-principles) calculations. Currently, this is the level (the minimum scale) for describing substances. The basis for first-principles calculations is electron wave function evolution by Schroedinger equation. We neither need additional assumptions for atoms interaction mechanism, nor any fitting formulas for interaction energy. However, this method has some disadvantages. Due to the complexity of formulating and solving equations for multielectron systems, the method allows to describe only simple systems - small perfect equilibrium structures.

The paper considers three types of potential. Several structure properties are obtained with the help of three different potential types - Morse potential, EAM and MEAM. The cohesive energy, lattice parameter, bulk modulus, shear modulus, Poisson ratio are calculated with help of three types of potentials. The aim of this work is to determine what potential is better suited for calculating properties of palladium.

\section{MATERIALS AND METHODS}

\section{Pair Potentials}

The first models of interatomic interaction were constructed with pair potentials. For such potentials the interaction energy of two particles depends only on the distance between them and does not depend on the environment. The energy of the system of atoms is given by:

$$
U\left(r_{1} \ldots r_{N}\right)=\frac{1}{2} \sum_{\substack{i, j=1 \\ i \neq j}}^{N} \varphi\left(r_{i j}\right)
$$

Here $\varphi\left(r_{i j}\right)$ - the energy of pair interaction between two atoms, $r_{i j}$ - the distance between them, $r_{1} \ldots r_{N}-$ the position of the point in which energy is determined.

Despite the simplicity of potential structure, the pair potential models are used to describe the properties of many substances. However, the pair potentials have significant disadvantages. The crystal lattices with low packing density (simple cubic) and covalent bonds is not stable for pair potentials. Pair potentials have bad transferability. Transferability is the ability of a potential to describe the substance under the different conditions.

\section{The Lennard-Jones potential}

One of the first potentials was proposed by Lennard-Jones:

$$
\varphi(r)=4 \varepsilon\left[\left(\frac{\sigma}{r}\right)^{12}-\left(\frac{\sigma}{r}\right)^{6}\right]
$$

Here, $\sigma$ is the equilibrium distance, when potential becomes zero, $\varepsilon-$ the depth of the potential well. The term with $\mathrm{r}^{-6}$ determines the attractive force. This term dominates at the long distance. The term with $\mathrm{r}^{-12}$ determines the repulsive force affecting on the short distance. 
The depth of the potential well ( $\varepsilon$ parameter) is determined from the binding energy and $\sigma$ parameter is determined from equilibrium lattice parameter. These two quantities can be determined experimentally or from theoretical calculations. Lennard-Jones potential is appropriate to describe inert gases and two atomic gas molecules. Occasionally it is used to describe metals, because it has a simple form and allows to simulate common phenomenon mechanisms.

\section{The Morse Potential}

The Morse potential has three adjustable parameters, therefore the potential is customized except binding energy and lattice parameter, on bulk modulus. It allows to use the Morse potential for describing metals with face centered cubic (fcc) lattice, base centered cubic (bcc) lattice and hexagonal close packed (hcp) lattice. The Morse potential has the following form:

$$
\varphi(r)=\varepsilon\left[\exp \left(-2 \alpha\left(r-r_{0}\right)\right)-2 \exp \left(-\alpha\left(r-r_{0}\right)\right)\right]
$$

Here, $r_{0}$ is the equilibrium distance, when potential becomes zero, $\varepsilon-$ the depth of the potential well. The graphics of Morse potential for different atom pairs are in Figure 2.

\section{Embedded Atom Method}

Embedded atom method was proposed by Dow and Baskes in 1987 [6], has been widely used for the simulation of matter at the atomic level.

EAM is based on density functional theory (DFT). The main DFT principles is the following. All bodies are known to consist of atoms and atoms their turn consist of nuclei and electrons. It is the distribution of nuclei and electrons charges form an electric field that is responsible for the structure of the matter and the processes in it. The deepest level of description of matter is the electron wave function. The wave function depends on space coordinates. The square of the wave function determines the probability of finding electron in this area.

The state of all electrons in substance is determined from wave function, which depends on the coordinates of all nuclei and all electrons of the substance. Even microscopic substance contains a very large number of atoms therefore determination of the wave function is almost impossible. Hohenberg and Kohn in 1964 suggested a simpler way for describing multielectron systems. They proved a theorem stating that the condition of the electrons in multielectron system can be described by means of functions of one variable. This variable is called the electron density and it is the function of the coordinates. In EAM the energy of one atom in the atomic structure is determined by the expression:

$$
\begin{gathered}
E_{i}=F(\rho)+\frac{1}{2} \sum_{i} \varphi_{i} \\
\rho_{i}=\sum_{j} \rho_{j}
\end{gathered}
$$

Here $\mathrm{F}$ - embedded function, $\rho \mathrm{i}$ - electron density at place i, $\varphi$ - pair interaction energy.

Thus the energy of each atom of the system is defined as the sum of the energy of the pair interaction of this atom with other atoms in the system plus embedded function. The embedded function differentiates EAM from pair potential and takes into account the influence of the environment. This function depends on the total electron density of all atoms, except one atom for which the energy is calculated. Consequently, for finding the total energy of the atom, it is necessary to define the following three functions: the energy of the pair interaction, electron density and embedded function. These functions are generally determined individually for different tasks. The functions can be obtained in two ways, either by quantum mechanical calculations or by fitting various approximation formulas. In the second case, the parameters of formulas are fitted to the experimental data.

Johnson developed an analytical embedded atom method and proposed the following formulas for three EAM functions [7]: 


$$
\begin{array}{r}
f(r)=f_{e} \exp \left[-\beta\left(\frac{r}{r_{e}}-1\right)\right], \quad r \leq r_{c} \\
\varphi(r)=\varphi_{e} \exp \left[-\gamma\left(\frac{r}{r_{e}}-1\right)\right], \quad r \leq r_{c} \\
F(\rho)=-E_{c B}\left[1-\frac{\alpha}{\beta} \ln \left(\frac{\rho}{\rho_{e}}\right)\right]\left(\frac{\rho}{\rho_{e}}\right)^{\alpha / \beta}-\Phi_{e}\left(\frac{\rho}{\rho_{e}}\right)^{\gamma / \beta}
\end{array}
$$

Here $\alpha=3 \sqrt{V_{a} B / \varepsilon_{0}}$

$B$ - bulk modulus, $V_{a}$ - atomic volume, $\rho_{e}=12 f_{e}$ and $\Phi_{e}=6 \varphi_{e}$. Parameters $\varphi_{e}, \beta, \gamma, r_{c}$ are determined by fitting to the characteristics of the material lattice parameter $a_{0}$, atomic volume $V_{a}$, binding energy $\varepsilon_{0}$, the formation energy of the vacancy $E_{v}$, bulk modulus $B$ and shear modulus $G$. [8]

The potentials developed for various atoms and compounds can be found in special databases in the Internet.

The EAM describes well the properties of many metals with face-centered cubic (fcc) and base-centered cubic (bcc) crystal lattice. However, structures with covalent bonds are described by this method much worse. The method describing such type of bonds will be considered below.

The problem of modeling the matter becomes much more complicated for systems consisting from different type of atoms. The EAM needs seven functions to describe a binary alloy. These are embedded functions and electron density for every type of atoms and three functions of pair interactions. Eleven functions are necessary for modeling systems with three types of atoms. In addition, it is necessary to identify a large number of parameters of these functions. One of the ways to determine the parameters is averaging. In this method the pair potential for the interaction between two different atoms is determined as the average of potentials of two atoms of the same type:

$$
\varphi_{A B}=\frac{1}{2}\left(\varphi_{A}+\varphi_{B}\right)
$$

Despite the fact that this method is not theoretically sound, the results of the calculations give good compliance with experimental data [9]. The EAM potential parts are presented on the Fig.3.

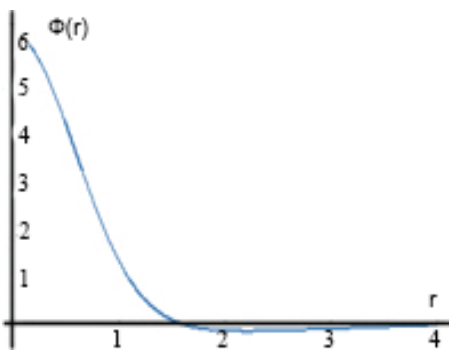

(a)

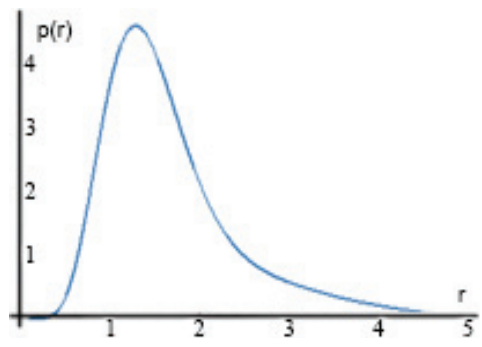

(b)

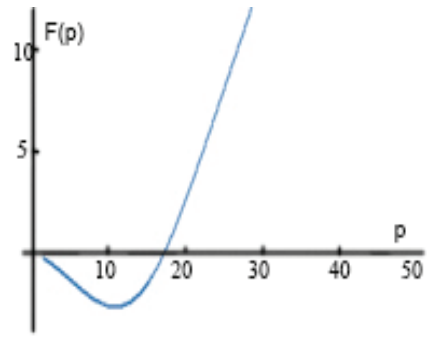

(c)

FIGURE 3. The pair interaction function (a), electron density function (b), embedded function (c) for palladium [10] 


\section{Modified Embedded Atom Method}

MEAM is developed on the basis of embedded atom method with some additions. The energy of atom in MEAM also consists of three functions (pair interaction, electron density and embedded functions), but each of them has a special form. Embedded function is determined from equation:.

$$
F=A E_{c} \frac{\rho}{\rho^{0}} \log \frac{\rho}{\rho^{0}}
$$

Here $\mathrm{A}$ is an adjustable parameter, Ec is the binding energy, $\rho$ is the electron density, $\rho 0$ is the electron density for a reference structure. The reference structure is a structure with atoms are on exact lattice points.

The form of electron density function is crucial for this method. In the EAM the electron density is spherically symmetric and the same is for all atoms in the system. Therefore, it cannot model a matter with different types of bonds. The electron density function in MEAM is determined as the sum of components. Each component is responsible for various directions of the bonds.

$$
\begin{gathered}
\left(\rho_{i}^{(0)}\right)^{2}=\left[\sum_{j \neq i} \rho_{j}^{a(0)}\left(R_{i j}\right)\right]^{2} \\
\left(\rho_{i}^{(1)}\right)^{2}=\sum_{\alpha}\left[\sum_{i \neq j} \frac{R_{i j}^{\alpha}}{R_{i j}} \rho_{j}^{a(1)}\left(R_{i j}\right)\right]^{2} \\
\left(\rho_{i}^{(2)}\right)^{2}=\sum_{\alpha, \beta}\left[\sum_{j \neq i} \frac{R_{i j}^{\alpha} R_{i j}^{\beta}}{R_{i j}^{2}} \rho_{j}^{a(2)}\left(R_{i j}\right)\right]^{2}-\frac{1}{3}\left[\sum_{j \neq i} \rho_{j}^{a(2)}\left(R_{i j}\right)\right]^{2} \\
\left(\rho_{i}^{(3)}\right)^{2}=\sum_{\alpha, \beta, \gamma}\left[\sum_{j \neq i} \frac{R_{i j}^{\alpha} R_{i j}^{\beta} R_{i j}^{\gamma}}{R_{i j}^{2}} \rho_{j}^{a(3)}\left(R_{i j}\right)\right]^{2}-\frac{3}{5} \sum_{\alpha}\left[\sum_{j \neq i} \frac{R_{i j}^{\alpha}}{R_{i j}} \rho_{j}^{a(3)}\left(R_{i j}\right)\right]^{2}
\end{gathered}
$$

Where $\rho_{\mathrm{i}}^{\mathrm{a}(\mathrm{h})}$ is atomic electron densities from $\mathrm{j}$ atom at a distance $\mathrm{R}_{\mathrm{ij}}$ from site $\mathrm{i} . \mathrm{R}_{\mathrm{ij}}{ }^{\alpha}$ is the $\alpha$ component of the distance vector between atoms I and $\mathrm{j}(\alpha=\mathrm{x}, \mathrm{y}, \mathrm{z})$. Different ways have been proposed to combine the partial electron densities.

Due to the ability to distinguish between different types of bonding, MEAM can be used to model most metals, semiconductors, alloys, oxides and even diatomic gases.

The second feature of the method is the possibility of considering the influence of the nearest neighboring atoms on the pair interaction function. The screening function is added to the pair interaction to take into account the nearest neighboring atoms.

$$
\begin{gathered}
S_{i j=\prod_{k \neq i, j} S_{i j k}} \\
S_{i j k}=f_{c}\left[\frac{C-C_{\min }}{C_{\max }-C_{\min }}\right] \\
\left\{\begin{array}{c}
f_{c}(x)=1, x \geq 1 \\
{\left[1-(1-x)^{4}\right]^{2}, 0<x<1} \\
0, x \leq 0
\end{array}\right.
\end{gathered}
$$

This function takes into account the influence of only the first nearest neighboring atoms and this method is called 1NN-MEAM. Occasionally, it is not sufficient for modeling some properties of matter. Therefore, the addition was suggested [9], it takes into account the influence of the second nearest neighboring atoms and this method is called 2NN-MEAM. Figure4 presents the principle of work of the screening function. 

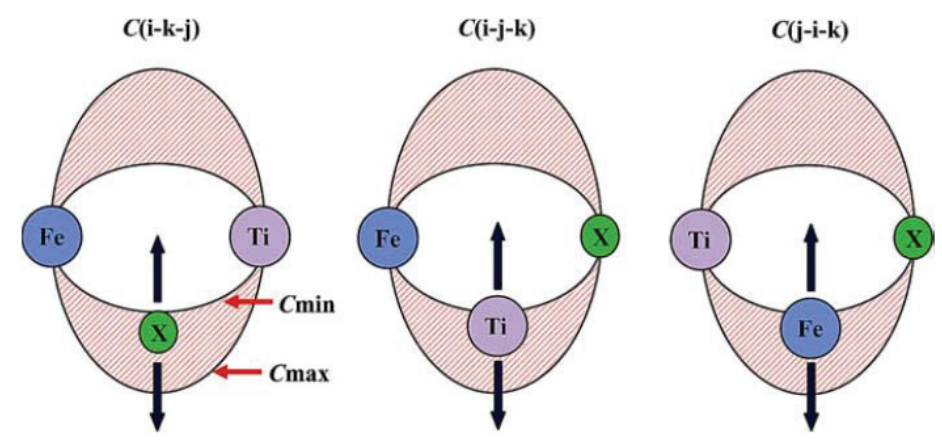

FIGURE 4. The scheme of screening function action [11].

The idea of the screening is following. If the atom $\mathrm{k}$ is inside of the ellipse defined by Cmin it is assumed that the atom k completely screens the ij interaction, between Cmax and Cmin the screening changes gradually. If the atom $\mathrm{k}$ is outside of the ellipse defined by Cmax the screening equal zero.

The parameters of the potential are known to fit from the experiment with the substance in certain conditions. Consequently, the potential with parameters derived from experimental characteristics of matter in volume (elastic coefficients, bulk modulus, binding energy, etc.) cannot predict the behavior of the surface atoms correctly. However, the 2-NN MEAM can model the substance in volume and on surface with the same parameters, because various bonding and environments can be determined. This potential has very good transferability.

\section{RESULTS AND DISCUSSION}

\section{Calculation of Palladium Elastic Properties}

We used the LAMMPS for calculation the elastic properties. LAMMPS is a program for molecular dynamics simulation developed in Sandia National Laboratories [12]. This program one the powerful MD simulator with many important applications for virtual constructing material. Crystal structure and Morse potential parameters are described in [4]. Morse parameters are $\varepsilon=0.471 \mathrm{eV}, \alpha=1.7 \AA^{-1}, \mathrm{r}_{0}=2.87 \AA$. The method of EAM potential creation are described in [10] and MEAM potential parameters are taken from LAMMPS meam library file. The values of bulk modulus, shear modulus and Poisson ratio for different potential types are presented in Table 1. Literature data are took from database [13].

TABLE 1. Palladium elastic properties.

\begin{tabular}{|c|c|c|c|c|}
\hline Property & Morse & EAM & MEAM & Literature \\
\hline Bulk Modulus, GPa & 196 & 199.12 & 195.7 & 180 \\
\hline Shear Modulus, GPa & 51 & 30 & 30 & 44 \\
\hline Poisson Ratio & 0.38 & 0.43 & 0.43 & 0.39 \\
\hline Lattice parameter, $\AA$ & 3.9 & 3.88 & 3.89 & 3.89 \\
\hline $\begin{array}{l}\text { Cohesion energy, } \\
\text { eV/atom }\end{array}$ & 3.58 & 3.91 & 3.91 & 3.89 \\
\hline
\end{tabular}

\section{CONCLUSION}

With the use of three different types of potentials - the Morse potential, EAM potential and MEAM potential, the following parameters of the structure of palladium are calculated: the lattice parameter, binding energy, elastic modulus, shear modulus, Poisson's ratio. The calculations are performed for ideal structures at zero temperature and pressure. In such circumstances, all potentials give similar values for the parameters, hence the Morse potential has the advantage, because to build it much easier than EAM and MEAM. All potentials have a problem with calculation of shear modulus. It is necessary to conduct a separate study to determine the cause 


\section{REFERENCES}

1. C. R. Weinberger and G. J. Tucker, Multiscale Materials Modeling for Nanomechanics (Springer, Switzerland, 2016), pp. 1-52.

2. A. M Ovrutsky, A. S. Prokhoda and M. S. Rasshchupkyna, Computational materials science (Elsevier, London, 2014), pp. 151-158.

3. Personal web page of Nadezhda Chistyakova. Available at: http://portal.tpu.ru:7777/SHARED/c/CHISTYAKOVANV/eng/Research/ElectrostaticField.

4. N. Chistyakova, Key Eng. Mater. 683 583-588 (2016).

5. W. Heitler and F. Z. London, Phys. 44 455-472 (1927)

6. M. S. Daw and M. I. Baskes, Phys Rev B 29 64-43 (1984).

7. R. A. Johnson, Phys. Rev. B 37 39-24 (1996).

8. Material Measurement Laboratory. Available at: http://www.ctcms.nist.gov/ cbecker/.

9. A. A. Nazarov and R. R. Mulyukov, Atomistic Simulation of Materials, Nanostructures and Nanotechnology Processes (Ufa, RIOBashGU, 2010), pp 25-27 (in Russian).

10. H. W. Sheng, M. J. Kramer, A. Cadien, T. Fujita and M. W. Chen, Phys.Rev.B. 83 118-134 (2011).

11. H.-K. Kim, W.-S. Jung and B.-J. Lee Acta Mater. 57 3140-3147 (2009).

12. LAMMPS Available at: http:// lammps.sandia.gov/.

13. Palladium Physical Properties. Available at: https://www.webelements.com/palladium/physics.html. 